Bio - grafía. Escritos sobre la Biología y su Enseñanza. ISSN 2027-1034

Edición Extraordinaria. p.p. 276 - 285

Memorias del IX Encuentro Nacional de Experiencias en Enseñanza de la Biología y la Educación Ambiental. IV Congreso Nacional de Investigación en Enseñanza de la Biología.

\title{
LAS INTERVENCIONES DIDÁCTICAS Y SU APORTE ALA CONSTRUCCIÓN DEL CONOCIMIENTO PROFESIONAL DE FUTUROS DOCENTES DE CIENCIAS NATURALES EN LA UNIVERSIDAD SURCOLOMBIANA
}

\author{
THE DIDACTIC INTERVENTIONS AND ITS CONTRIBUTION TO THE \\ CONSTRUCTION OF THE PROFESSIONAL KNOWLEDGE OF FUTURE \\ TEACHERS OF NATURAL SCIENCES AT THE SURCOLOMBIAN UNIVERSITY
}

\author{
Bahamón Calderón, Edinsson ${ }^{1}$ \\ Castrillón Andrade, Jennifer ${ }^{1}$ \\ Amórtegui Cedeño, Elías Francisco ${ }^{2}$ \\ Mosquera, Jonathan Andrés ${ }^{3}$.
}

\section{RESUMEN}

A continuación presentamos resultados preliminares de la sistematización y el análisis de las intervenciones didácticas realizadas por futuros docentes de ciencias naturales, sobre las temáticas sistema digestivo y sistema muscular, al interior de un curso sobre Didáctica de las Ciencias al interior de la Licenciatura en Ciencias Naturales: Física, Química y Biología de la Universidad Surcolombiana (Neiva- Colombia). Este estudio adelantado durante el año 2016, aborda en concreto las contribuciones a la construcción del Conocimiento Profesional, de los espacios académicos en la etapa de la formación inicial docente. La perspectiva metodológica es de tipo cualitativa, con enfoque interpretativo, empleando el método de análisis de contenido a través del software Atlas $\mathrm{Ti}$ 7.0, para hacer revisión, categorización y análisis de las producciones escritas de los futuros docentes. Como resultados principales, destacamos que en las planeaciones elaboradas por los estudiantes-docentes, se evidencian planteamientos en relación a las categorías enseñanza, finalidades de enseñanza, estrategias de enseñanza, dificultades de aprendizaje y rol docenteestudiante, que permiten la configuración de un conocimiento del profesor de referencia.

PALABRAS CLAVE: Conocimiento Profesional del Profesor, Conocimiento Didáctico del Contenido, Formación Docente.

\section{ABSTRACT}

Next, we present preliminary results of the systematization and analysis of the didactic interventions carried out by future teachers of natural sciences, on the subject's

\footnotetext{
1 Estudiantes de Licenciatura en Ciencias Naturales: Física, Química y Biología. Universidad Surcolombiana. bahamon12@gmail.com niye 11@hotmail.com. Grupo de Investigación Conocimiento Profesional del Profesor de Ciencias

2 Docente de Planta Tiempo Completo. Licenciatura en Ciencias Naturales: Física, Química y Biología. Universidad Surcolombiana. elias.amortegui@usco.edu.co Grupo de Investigación Conocimiento Profesional del Profesor de Ciencias

3 Docente de Cátedra e Investigador. Licenciatura en Ciencias Naturales: Física, Química y Biología. Universidad Surcolombiana. jonathan.mosquera@usco.edu.co . Grupo de Investigación Conocimiento Profesional del Profesor de Ciencias
} 
Bio - grafía. Escritos sobre la Biología y su Enseñanza. ISSN 2027-1034

Edición Extraordinaria. p.p. 276 - 285

Memorias del IX Encuentro Nacional de Experiencias en Enseñanza de la Biología y la Educación Ambiental. IV Congreso Nacional de Investigación en Enseñanza de la Biología.

digestive system and muscular system, within a course on Didactics of the Sciences within the Bachelor in Natural Sciences: Physics, Chemistry and Biology of the Surcolombian University (Neiva- Colombia). This study, advanced in 2016, specifically addresses the contributions to the construction of Professional Knowledge, of academic spaces in the initial teacher education stage. The methodological perspective is qualitative, with an interpretative approach, using the method of content analysis through Atlas Ti 7.0 software, to review, categorize and analyze the written productions of future teachers. As main results, we highlight that in the planning developed by student-teachers, there are evidences regarding teaching categories, teaching purposes, teaching strategies, learning difficulties and teacher-student role, which allow the configuration of a knowledge Of the reference teacher.

KEY WORDS: Knowledge professional teacher, didactic knowledge of content, teacher training.

\section{INTRODUCCIÓN}

Este estudio está enmarcado en la perspectiva del Conocimiento Profesional del Profesor de Ciencias, asumiendo que los docentes construyen un conjunto de saberes y prácticas que les diferencia de otros profesionales (Bromme, 1988; Valbuena, 2007; Tardif \& Lessar, 2014) y les permite enseñar de manera efectiva. En términos generales, éste, se constituye a partir de cuatro grandes componentes: el Conocimiento del Contexto, el Conocimiento de la Materia (Subjet Matter) que se enseña, el Conocimiento Pedagógico y el Conocimiento Didáctico del Contenido-CDC(Pedagogical Content Knowledge), del cual la diversa literatura especializada ha consensuado que se configura como el núcleo del Conocimiento del Profesor (Shulman 1986, 1987; Grossman, 1990; Carlsen, 1999; Magnusson, Krajcik \& Borko, 1999; Gess-Newsome, 1999; Perafán, 2005, Abell, 2008, Park \& Oliver, 2008; Berry, Loughran \& Van Driel, 2008; Nilsson, 2008; Rollnick et al, 2008; Park \& Oliver; 2008, Park \& Chen, 2012; Gess-Newsome, 2015). Este CDC se compone a su vez de varios elementos de diversas fuentes y naturaleza, tales como las estrategias de enseñanza, las finalidades de enseñanza, el conocimiento sobre las ideas previas del alumnado, la evaluación del aprendizaje, entre otros.

De acuerdo con Martínez y Molina (2009), los estudios sobre el Conocimiento Profesional en el contexto de profesores de Ciencias Naturales en educación básica, son escasos. Ahora bien, para el caso del departamento del Huila, se destacan los trabajos de Amórtegui et al. (2015) con docentes en formación en el contexto de su Práctica Pedagógica, así mismo Mosquera y Amórtegui (2017), abordan un estudio enfocado a los docentes principiantes graduados en un periodo no mayor a 3 años de la Licenciatura en Ciencias Naturales de la Universidad Surcolombiana.

De esta manera, siendo la Universidad Surcolombiana la única Institución de Educación Superior del Departamento del Huila, encargada de la formación inicial de profesores de ciencias naturales, los estudios sobre la construcción del Conocimiento del Profesor son incipientes, sin embargo, la tendencia en la formación se acerca a una postura constructivista. Resaltamos la compilación de Amórtegui \& Cuellar (2014) como una primera sistematización de la práctica docente en la formación inicial del 
Bio - grafía. Escritos sobre la Biología y su Enseñanza. ISSN 2027-1034

Edición Extraordinaria. p.p. 276 - 285

Memorias del IX Encuentro Nacional de Experiencias en Enseñanza de la Biología y la Educación Ambiental. IV Congreso Nacional de Investigación en Enseñanza de la Biología.

profesorado huilense. Con base en lo anterior nos planteamos como objetivo principal caracterizar el aporte de las intervenciones de aula en la construcción del Conocimiento del Profesor en formación de Ciencias Naturales en la Universidad Surcolombiana.

\section{METODOLOGÍA}

Esta investigación se define desde un enfoque cualitativo, de acuerdo con Álvarez y Jurgenson (2003), este enfoque considera un diseño de la investigación flexible, en donde el investigador ve el escenario y a las personas desde una perspectiva holística, en este caso los futuros profesores de Ciencias Naturales, además dichas personas, los escenarios o los grupos no son reducidos a variables, sino que son considerados como un todo. La población de estudio, comprende un grupo de 26 estudiantesdocentes, de los cuales por consentimiento voluntario, 13 aceptaron participar en el proyecto. Los futuros docentes, tienen entre 20 y 25 años de edad, pertenecen a estratos socio-económicos 1 y 2 , y cursaron espacios académicos sobre Didáctica de las Ciencias Naturales durante el segundo semestre del 2015 y el I primer semestre del 2016. La principal actividad de este último espacio académico consiste en que los futuros docentes, realicen planeaciones de clases e intervenciones didácticas de una temática de ciencias naturales (Biología, Química, Física) con estudiantes de educación básica secundaria de alguna institución educativa oficial de la ciudad de Neiva.

Las fuentes de información son las producciones escritas de los estudiantes-docentes, sobre las cuales se procedió a realizar el análisis de contenido. La intervención es planificada a través de tres entregas habitualmente, para este caso se sistematizó y analizó la tercera entrega. En el análisis se sigue lo propuesto por Amórtegui y Correa (2012), ubicando las unidades de información (UI) de cada fuente, y se tuvo en cuenta las categorías del CPP y del CDC, establecidas por Valbuena et al. (2009) y por Park y Chen (2012). El proceso anterior de sistematización, se hizo con apoyo del software Atlas. Ti 7.0.

\section{RESULTADOS}

En este apartado nos referimos específicamente a la sistematización de la intervención (tercera entrega) que realizaron los futuros docentes, para esto, mostramos una red de hallazgos para cada caso, las principales categorías y tendencias encontradas. Además mostramos evidencias y un análisis sobre estos.

\section{C1: Enseñanza del sistema digestivo.}


Bio - grafía. Escritos sobre la Biología y su Enseñanza. ISSN 2027-1034

Edición Extraordinaria. p.p. $276-285$

Memorias del IX Encuentro Nacional de Experiencias en Enseñanza de la Biología y la Educación Ambiental. IV Congreso Nacional de Investigación en Enseñanza de la Biología.

Podemos destacar que para esta tercera entrega, los futuros docentes reconocieron 5 grandes categorías: ENSEÑANZA, FINALIDADES DE ENSEÑANZA, ESTRATEGIAS

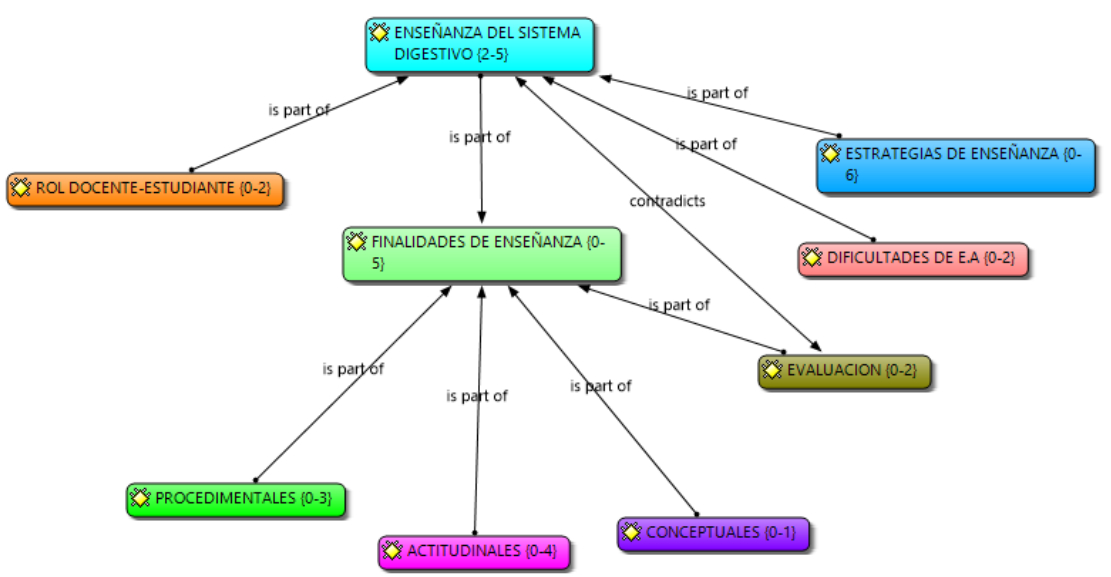

DE ENSEÑANZA, DIFICULTADES, Y ROL DOCENTE-ESTUDIANTE (Ver Figura 1).

Figura 1. Categorías del CPP halladas en el Caso 1

De este primer caso, destacamos dos categorías principalmente. Frente al ESTRATEGIAS DE ENSEÑANZA (Figura 2), el docente en formación, afirma que:

Cl: 1:11 [Haciendo referencia al contexto del alumnado] “...es indispensable haber elaborado con anterioridad el diario de campo y su respectiva interpretación; esta herramienta es importante, puesto que, permite analizar, cambiar, mejorar o quitar aspectos o actividades que puedan beneficiar o no el desarrollo de clase..."

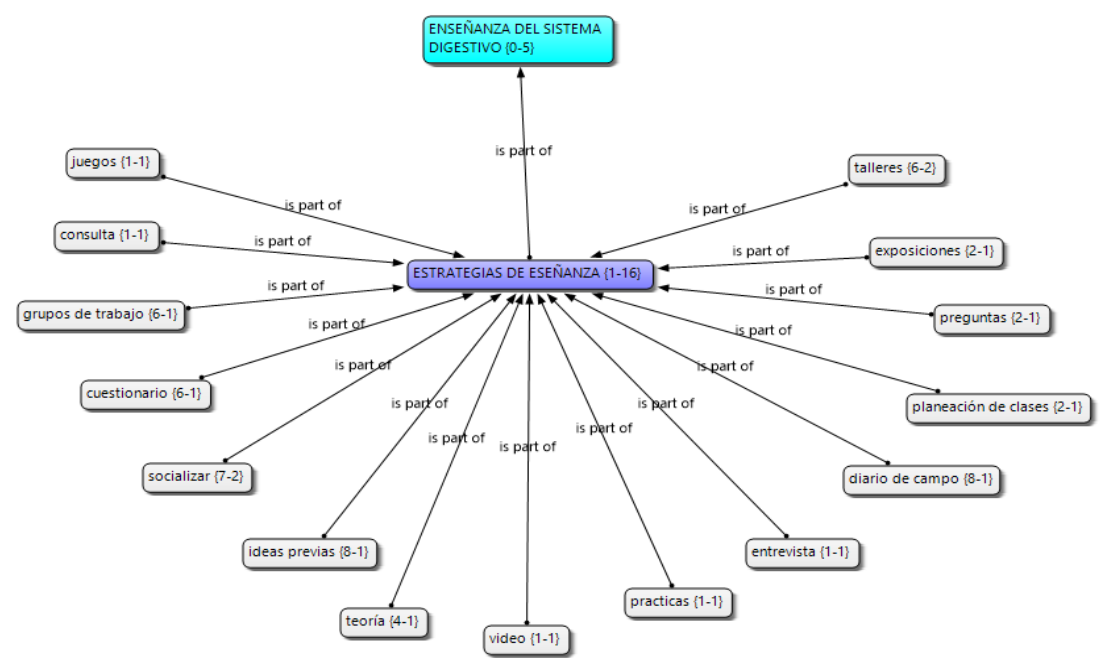

Figura 2. Categoría Estrategia de Enseñanza para el Caso 1

Aquí, el futuro docente muestra diferentes estrategias que utilizó para el proceso de enseñanza-aprendizaje de las ciencias naturales más desde una perspectiva 
Bio - grafía. Escritos sobre la Biología y su Enseñanza. ISSN 2027-1034

Edición Extraordinaria. p.p. 276 - 285

Memorias del IX Encuentro Nacional de Experiencias en Enseñanza de la Biología y la Educación Ambiental. IV Congreso Nacional de Investigación en Enseñanza de la Biología.

constructivista. Para este docente en formación, es fundamental tener estrategias de enseñanza en una intervención de aula, dado que facilitan el aprendizaje por parte de los estudiantes, generando motivación y facilitando la aproximación a la ciencia. Para Castro y Valbuena (2007), el aprendizaje debe posibilitar a los estudiantes tener una mejor comprensión de lo que ocurre en el contexto científico, de tal forma que las estrategias son esenciales para lograr incidir notablemente en el desarrollo de actitudes positivas hacia lo vivo. En la definición de las estrategias se hace necesario entonces conocer los conceptos previos del estudiantado, de acuerdo con Jara, Cuetos y Serna (2015).

La segunda categoría que destacamos, es ROL DOCENTE-ESTUDIANTE (Figura 3), el docente en formación reconoce que:

Cl: 1:12 [Haciendo referencia al contexto del alumnado] “...Me di cuenta de algo importantísimo $y$ es que, para tener unos estudiantes motivados $y$ concentrados en las clases, es necesario pensar desde el punto de vista de ellos, con el fin de identificar que quisieran ellos recibir del maestro en las diferentes circunstancias que se puedan presentarse dentro y fuera del aula de clases..."

En esta ocasión el docente en formación, plantea una relación horizontal con sus estudiantes, concibiendo que el proceso de enseñanza y aprendizaje, es mediado entre las dos partes y que la reflexión por parte del profesor, es esencial para poder replantear actividades o valorar el papel que los dos actores están desempeñando. Esta postura guarda relación con planteamientos más constructivistas en el campo de la didáctica, pues como afirman Rodrigo et al. (1999), las actividades de enseñanzaaprendizaje incluye diversos roles para el docente y los estudiantes, de acuerdo con el enfoque y con el tipo de trabajo que se esté realizando, estableciendo que desde un enfoque tradicional, el profesor actuaría como expositor omnisciente, en donde el papel del estudiante sería el de escuchar y redescubrir las teorías; mientras que en enfoques más abiertos, se permitiría una mayor participación de los estudiantes siendo protagonistas del proceso y el docente asumiría el rol de guía y orientador de procesos.

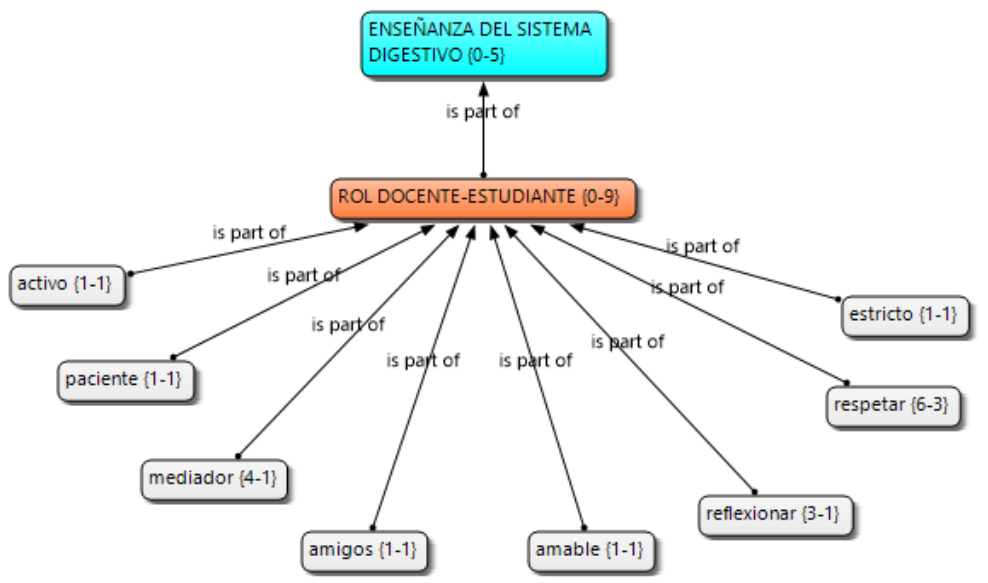

Figura 3. Categoría Rol Docente para el Caso 1 
Bio - grafía. Escritos sobre la Biología y su Enseñanza. ISSN 2027-1034

Edición Extraordinaria. p.p. 276 - 285

Memorias del IX Encuentro Nacional de Experiencias en Enseñanza de la Biología y la Educación Ambiental. IV Congreso Nacional de Investigación en Enseñanza de la Biología.

Una categoría que busca destacar el docente en formación son las relaciones interpersonales de los estudiantes, donde se evidencien los valores y se posibilita un acercamiento para comprender las singularidades de los estudiantes contribuyendo a las finalidades de enseñanza en cuanto a las actitudes de los estudiantes, además el profesor se configura como una guía o mediador del aprendizaje.

\section{C2: Enseñanza del sistema muscular.}

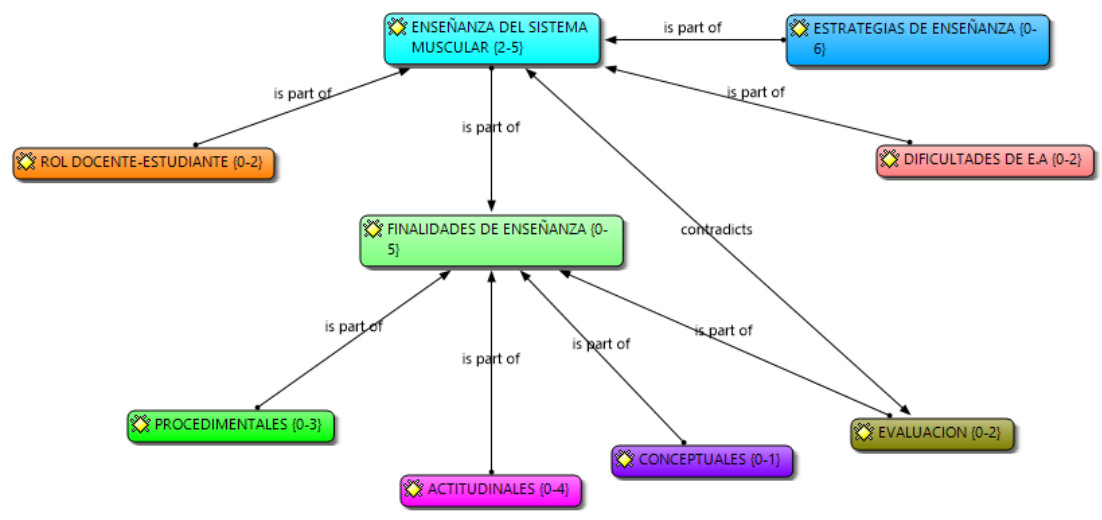

Figura 4. Categorías del CPP para el Caso 2

En el segundo caso, en la planeación del futuro docente, se reconocen 5 grandes categorías: ENSEÑANZA, FINALIDADES DE ENSEÑANZA, ESTRATEGIAS DE ENSEÑANZA, DIFICULTADES, Y ROL DOCENTE-ESTUDIANTE (Ver Figura 4).

Resaltamos para el Caso 2, la categoría DIFICULTADES DE ENSEÑANZA, pues aquí el estudiante-docente afirma que:

CI: $1: 4$ [Haciendo referencia al contexto del alumnado] "...encontramos que una de las principales dificultades de enseñanza y aprendizaje sobre el tema del Sistema Muscular es que no tienen claro su definición y funcionamiento, es decir que no lo asocian al movimiento y estabilidad del cuerpo, otra dificultad que se observo fue el hecho de que no mencionan por ningún lado los tendones, siendo uno de los componentes principales del sistema muscular, por esta razón, se deduce que los niños solo lo asumen hacia los músculos...." 
Bio - grafía. Escritos sobre la Biología y su Enseñanza. ISSN 2027-1034

Edición Extraordinaria. p.p. $276-285$

Memorias del IX Encuentro Nacional de Experiencias en Enseñanza de la Biología y la Educación Ambiental. IV Congreso Nacional de Investigación en Enseñanza de la Biología.

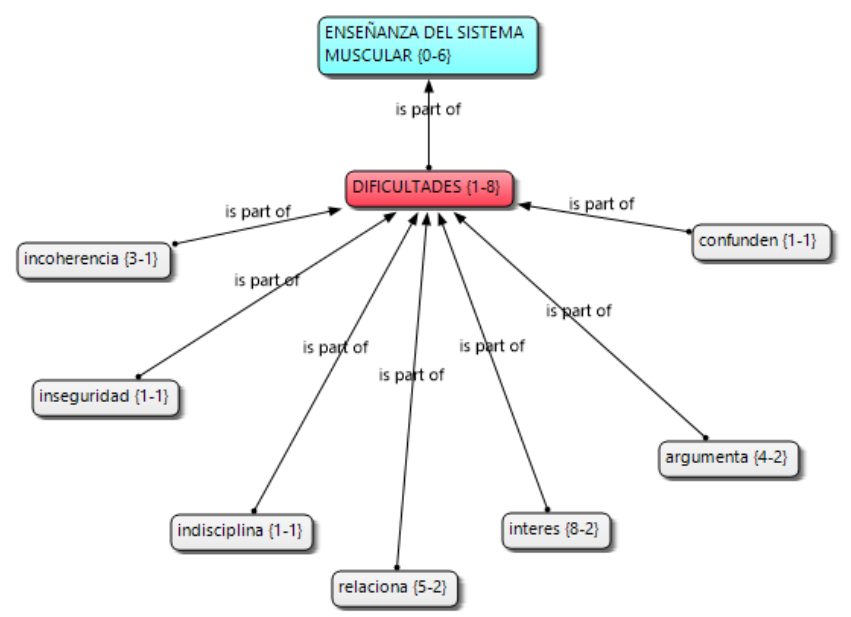

Figura 5. Categoría Dificultades de Aprendizaje para el Caso 2

En este caso, el docente considera que dentro de las principales dificultades posibles en la enseñanza-aprendizaje del sistema muscular, está la falta de relacionar conceptos por parte de los estudiantes y sus propias experiencias. Así mismo manifiesta la poca capacidad de argumentación, por tal motivo tiende a confundirse y sin justificar sus ideas, prefieren mantener al margen del proceso educativo. Esta valoración que hace el profesor, es indispensable, ya que conoce las dificultades de los estudiantes durante las intervenciones de aula, permite redefinir actividades, estrategias de enseñanza y evaluar de manera holística las condiciones que están permitiendo o afectando el aprendizaje. Según Amórtegui et al, (2015), los docentes en formación tienden a valorar los aspectos personales y afectivos del estudiantado, dado que las ideas previas y concepciones que se forjan en el marco social y familiar que envuelve al alumno, ejerce un papel muy importante en la vida académica de los mismos, influyendo en el funcionamiento cognitivo y de motivación, por tanto, es importante conocer algunas características no solo cognitivas, sino sociales del alumnado.

De igual forma, estas dificultades planteadas por el futuro docente, no son ajenas a la luz de la teoría de la didáctica de las ciencias, dado que como considera Jiménez (2003), el aprendizaje de las ciencias no puede ser concebido sólo en términos cognitivos; se debe contar con el desarrollo afectivo, es decir, debemos tener en cuenta no sólo lo que los estudiantes piensan, sino también lo que sienten. En atención a esto, autores como Mellado (2003) y Borrachero (2015) plantean que es importante que el docente en formación llegue a comprender las emociones de sus estudiantes a través del ejercicio de la franqueza y flexibilidad emocional, permitiéndole mirar su historia dentro de la de sus alumnos.

Por otra parte, hablamos de FINALIDADES DE ENSEÑANZA DE TIPO ACTITUD INAL, en la cual aporta a la formación como docente. 
Bio - grafía. Escritos sobre la Biología y su Enseñanza. ISSN 2027-1034

Edición Extraordinaria. p.p. 276 - 285

Memorias del IX Encuentro Nacional de Experiencias en Enseñanza de la Biología y la Educación Ambiental. IV Congreso Nacional de Investigación en Enseñanza de la Biología.

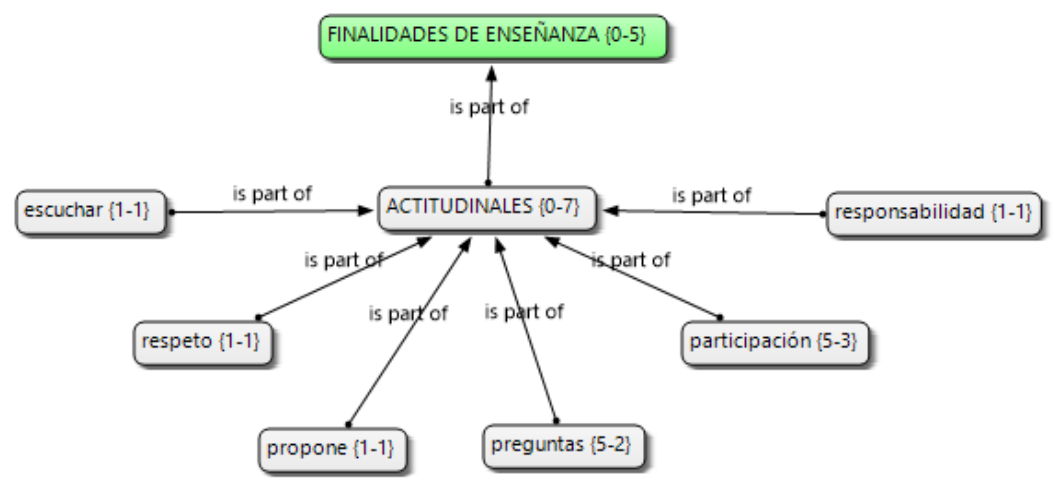

Figura 6. Categoría Finalidades de Enseñanza conceptuales para el Caso 2

CI: 1:4 [Haciendo referencia al contexto del alumnado] "...En general el grupo estuvo atento y respondieron satisfactoriamente a las explicaciones dadas, el tiempo fue preciso y se logró abordar el tema para esa primera clase".

En esta finalidad encontramos la necesidad de reforzar sus actitudes, que corresponde desde una perspectiva más constructivista, según Valbuena (2007), con una enseñanza de la Biología que consista en la organización de objetivos y contenidos formativos, y la implementación de actividades orientadas al aprendizaje significativo, al desarrollo metacognitivo y a la construcción de conocimiento escolar, principalmente al tener en cuenta las características del alumno, del conocimiento biológico y del contexto.

\section{CONCLUSIONES}

Se evidencia en las producciones de los futuros docentes concepciones de tipo constructivistas, valorando que en el proceso de enseñanza y aprendizaje de las ciencias, participan diferentes factores y que el protagonismo de una secuencia de aula, es compartido entre el profesor y los estudiantes. Esta progresión ha sido revisada por otros autores para la formación de profesores de ciencias, validando la importancia de los espacios formativos en la configuración de saberes y conocimientos propios del docente. Aquí la reflexión del docente se estructura como eje de su propio proceso formativo, y en donde el análisis y uso del contexto se convierte en un elemento esencial en el aula.

Finalmente, los docentes en formación, consideran que el diseño y planificación de estrategias de enseñanza, es un proceso crucial en el aprendizaje de las ciencias, pues permite motivar y despertar emociones en el estudiante, recabando en ideas previas que en conjunto con el conocimiento científico, pueden permitir la configuración de un conocimiento escolar. Estos hallazgos, nos permite plantear que el conjunto de elementos que abordan estos estudiantes-docentes al interior del espacio formativo, junto con la experiencia que les da la intervención didáctica y las demás actividades en el aula, les permite construir un conocimiento profesional propio y de referencia, particularmente construido en el contexto educativo y social del departamento del Huila. 
Bio - grafía. Escritos sobre la Biología y su Enseñanza. ISSN 2027-1034

Edición Extraordinaria. p.p. $276-285$

Memorias del IX Encuentro Nacional de Experiencias en Enseñanza de la Biología y la Educación Ambiental. IV Congreso Nacional de Investigación en Enseñanza de la Biología.

\section{REFERENCIAS BIBLIOGRÁFICAS}

Abell, S. (2008). Twenty Yaers Later: Does Pedagogical Content Knowledge remain a useful idea? International Journal of Science Education.30 (10), 1405-1416.

Amórtegui, E. y Cuéllar, Z. (2014). Experiencias en la enseñanza de las ciencias naturales y formación inicial de maestros en el Departamento del Huila, Neiva: Editorial Universidad Surcolombiana.

Amórtegui, E., Mosquera, J., Quiroga, A., Dussan, G., Bernal, J. Dussan, N., Rodríguez, H. y Huependo, Y. (2015). Construcción del Conocimiento Profesional del Profesor de Ciencias Naturales en el Contexto de la Práctica Pedagógica en la Unifraersidad Surcolombiana, Caso Mario, Resultados Preliminares. Revista Bio-grafía: Escritos sobre biología y su enseñanza, pp.1204- 1211.

Mosquera, J. y Amortegui, E. (2017). Construcción del conocimiento del profesor principiante de ciencias naturales. Memorias del III Encuentro Nacional de Grupos de Investigación en Educación. Neiva, Colombia.

Barnett, J. y Hodson, D. (2001). Pedagogical context knowledge: Toward a fuller understanding of what good science teachers know. Science Education, 85(4), pp. 426-453.

Berry, A., Loughran, J. y Van Driel, J. H. (2008). Revisiting the Roots of Pedagogical Content Knowledge. International Journal of Science Education, 30:10, 1271 1279.

Bromme, R. (1988). Conocimientos profesionales de los profesores. Enseñanza de las Ciencias, 6 (1), 19-29.

Carlsen, W. (1999). Domains of Teacher Knowledge. In: Gess-newsome, J. y Lederman, N. (Eds.). Examining Pedagogical Content Knowledge. The Construct and its Implications for Science Education. Dordrecht, Boston, London: Kluwer Academic Publishers. pp. 133-144.

Castro, J y Valbuena, E (2007). ¿Qué biología enseñar y cómo hacerlo? Hacia una resignificación de la Biología escolar. Tecné Episteme y Didaxis. 22. 126-145.

Gess-newsome, J. (1999). Pedagogical Content Knowledge: An introduction and orientation. In: Gess-newsome, J. y Lederman, N. (Eds.). Examining Pedagogical Content Knowedge. The Construct and its Implications.

Gess-newsome, J. (2015). A model of teacher professional knowledge and skill incluiding PCK. In: Berry, A., Friedrichsen, P., y Loughran, J. Re-examining Pedagogical Content Knowledge in Science Education. New York: Routledge.

Jara, D., Cuetos, M.J. y Serna, A.I (2015). Didáctica de las ciencias naturales en educación primaria. La Rioja: Universidad Internacional de la Rioja.

Grossman, P. (1990). The Making of a Teacher. Theacher Knowledge and Teacher Education. New York: Teachers College, Columbia University.

Magnusson, S., Krajcik, J. yBorko, H. (1999). Nature, Sources, and Development of Pedagogical Content Knowledge for Science Teaching. In: Examing Pedagogical 
Bio - grafía. Escritos sobre la Biología y su Enseñanza. ISSN 2027-1034

Edición Extraordinaria. p.p. 276 - 285

Memorias del IX Encuentro Nacional de Experiencias en Enseñanza de la Biología y la Educación Ambiental. IV Congreso Nacional de Investigación en Enseñanza de la Biología.

Content Knowledge. The Construct and its Implications for Science Education. Dordrecht, Boston, London: Kluwer Academic Publishers. pp. 95-132.

Martínez, C. y Molina, A. (2009). El conocimiento profesional de los profesores de ciencias de primaria sobre el conocimiento escolar en el distrito capital: un problema de investigación. Memorias I congreso Nacional de investigación en educación en ciencias y tecnología.

Nilsson, P. (2008). Teaching for Understanding: The complex nature of pedagogical content knowledge in preservice education. International Journal of Science Education, 30 (10), Pp 1281 - 1299.

Park, S. y Chan, Y. (2012). Mapping out the Integration of the Components of Pedagogical Content Knowledge (PCK): Examples from High School Biology Class-rooms. Journal of Research in Science Teaching. 1-20

Park, S. y Oliver, S. (2008). Revisiting the conceptualisation of Pedagogical Con-tent Knowledge (PCK). Research in Science Education, 38, 261-284.

Perafán, A. (2005). La epistemología del profesor sobre su propio conocimiento profesional. Bogotá: Universidad Pedagógica Nacional.

Rodrigo, M., Morcillo, J., Borges, R., Calvo, A., Cordeiro, N., García, F. y Raviolo, A. (1999). Concepciones sobre el trabajo práctico de campo (TPC): Una aproximación al pensamiento de los futuros profesores. Revista Complutense de Educación, 10 (2), 261-285.

Rollnick, M., Bennett, J., Rhemtula, M., Dharsey, N. y Ndlovu, T. (2008). The Place of Subject Matter Knowledge in Pedagogical Content Knowledge: A case study of South African teachers teaching the amount of substance and chemical equilibrium. International Journal of Science Education, 30 (10), 1365 - 1387.

Shulman, L. (1986). Those who understand: Knowledge growth in teaching. Educational Researcher, 15 (2), 4-14.

Tardif, M. (2004). Los saberes del docente y su desarrollo profesional. Traducción de Pablo Manzano. Madrid: Narcea.

Tardif, M. y Lessard, C. (2014).O ofício de profesor. História, perspectivas e desafíos internacionais. RJ: Editora Vozes.

Torrente, M., Guevara, W. y Amórtegui, E. (2014). Concepciones acerca de la enseñanza-aprendizaje de las ciencias naturales de futuros docentes. En: Amórtegui, E. y Cuéllar, Z. (2014). Experiencias en la enseñanza de las ciencias naturales y formación inicial de maestros en el Departamento del Huila. Neiva: Editorial Universidad Surcolombiana. 